\title{
MECHANISMS OF YELLOW AND RED PHOTOLUMINESCENCE IN WURTZITE AND CUBIC GaN
}

M. Godlewski ${ }^{a}$, T. Suski ${ }^{b}$, I. GRzegori ${ }^{b}$, S. Porowski ${ }^{b}$, R. LANGeR ${ }^{c}$, A. Barski ${ }^{c}$, J.P. Bergman ${ }^{d}$, B. Monemar ${ }^{d}$, E.M. Goldys ${ }^{e}$ AND M.R. PHILlips $f$

${ }^{a}$ Institute of Physics, Polish Academy of Sciences Al. Lotników 32/46, 02-668 Warsaw, Poland

${ }^{b}$ High Pressure Research Center, Polish Academy of Sciences Sokolowska 29, 01-142 Warsaw, Poland ${ }^{c}$ CEA-Grenoble, DRFMC/SP2M, 17 rue des Martyrs, 38054 Grenoble, France ${ }^{d}$ Dept. Phys. \& Meas. Technol., Linköping Univ., 58183 Linköping, Sweden ${ }^{e}$ Semicond. Sci. \& Technol. Lab., Macquarie Univ. North Ryde, 2109 NSW, Australia

${ }^{f}$ Microstructural Analysis Unit, University of Technology, Sydney, Australia

The origin of two "deep" photoluminescence (PL) emissions observed in wurtzite (yellow PL) and cubic (red PL) GaN is discussed. PL and time-resolved PL studies confirm donor--acceptor pair character of the yellow band in wurtzite $\mathrm{GaN}$ and point to participation of shallow donors in this emission. A similar PL mechanism is proposed for the red emission of cubic GaN. We further show a puzzling property of both yellow and red PLs. Both yellow and red emissions show spatial homogeneity and are only weakly dependent on surface morphology.

PACS numbers: $61.16 . \mathrm{Ch}, 61.72 . \mathrm{Ff}, 71.55 . \mathrm{Eq}$

The characteristic property of bulk samples and epilayers of GaN is the presence of yellow photoluminescence (PL) emission band with the maximum at about $2.2 \mathrm{eV}$. The origin of this $\mathrm{PL}$, which is observed in practically all samples of wurtzite structure, grown by different techniques [1], is still disputed. Two conflicting models were proposed to explain the yellow PL [1-3] within the framework of the donor-acceptor pair (DAP) recombination mechanism. The yellow PL has been related to either DAP recombination of shallow donors and deep acceptors $[1,2]$, or to a deep donor-shallow acceptor related DAP process [3]. Recently, a successful growth of cubic phase GaN epilayers was achieved [1]. In addition to excitonic edge emissions, these epilayers show red $\mathrm{PL}$, with the maximum at about $2 \mathrm{eV}$. The origin of this PL remains unknown.

*fax: 48-22-8430926, e-mail: godlew@ifpan.edu.pl 
In this work we investigate the microscopic mechanisms of two "deep" PL emissions, the yellow PL of wurtzite GaN and the red PL, observed in cubic GaN. The results of PL and PL kinetics experiments are discussed. The influence of surface morphology on the two "deep" PL emissions is also studied based on the results of atomic force (AFM), scanning electron (SEM) microscopies and cathodoluminescence (CL) imaging. In our experiments we used either bulk GaN samples or $\mathrm{GaN}$ epilayers of wurtzite or zinc blende structure. GaN epilayers were grown on Si substrate by molecular beam epitaxy (MBE) in CEA Grenoble. Bulk GaN samples, highly conductive $n$-type (as-grown undoped samples) or highly resistive $\mathrm{Mg}$-doped, were grown in High Pressure Research Center of the Polish Academy of Sciences, Warsaw.

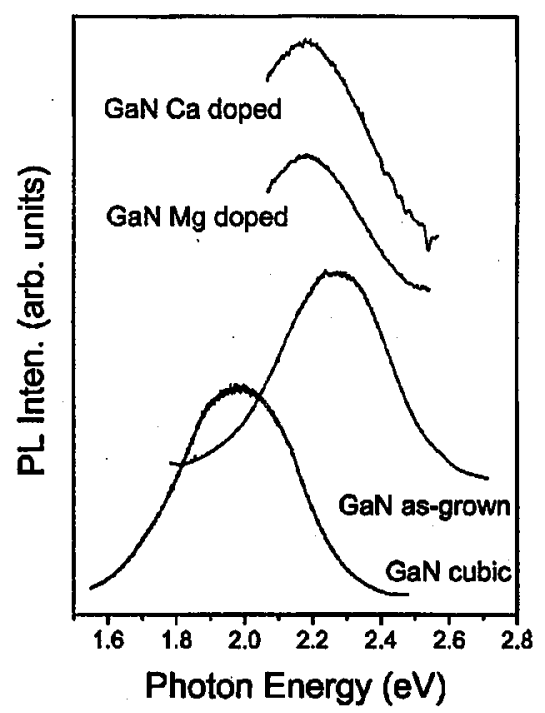

Fig. 1. Low energy part of the photoluminescence spectra taken at $2 \mathrm{~K}$ for three bulk GaN samples and one cubic GaN epilayer. As-grown and two acceptor compensated (in melt) bulk samples were studied.

In Fig. 1 we show the low energy part of the PL spectrum for wurtzite and cubic GaN materials. The PL spectrum of wurtzite GaN is dominated by the yellow PL band, which has the maximum at $2.2 \mathrm{eV}$ or $2.3 \mathrm{eV}$ (for degenerate $n$-type samples). The PL kinetics of the yellow PL and of "edge" excitonic transitions (not shown in Fig. 1) was measured. In GaN epilayers of cubic structure the PL decay time could be measured only for "edge" PL, since in most of the cubic epilayers the red PL was too weak. Whereas the PL decay times for excitonic and near band gap DAP and free-to-bound (FB) emission processes for both wurtzite and cubic GaN are between $100 \mathrm{ps}$ (for free and bound excitons) and $1 \mathrm{~ns}$ (for "shallow" DAP and FB transitions), the decay time of the yellow PL varies. A fast decay of the yellow PL (in ns time range) is observed in as-grown bulk samples, which were heavily $n$-type. For these samples the decay time is energy-independent and is associated 
by us with the degenerate $n$-type conductivity. Formation of donor band and fast migration of electrons from one to another donor site is then expected [4]. For such samples the yellow band maximum is observed at a slightly larger energy (see Fig. 1) in close analogy to FB transitions.

In compensated bulk samples and in wurtzite $\mathrm{GaN}$ epilayers the decay time of the yellow PL becomes energy dependent (shorter decay times are observed at the high-energy wing of the PL emission) and is much longer; up to $500 \mu \mathrm{s}$ for the slow component of the decay. Both the value and the energy dependence of the decay time are characteristic of the DAP transitions with a spatial distribution of D-A pairs. Thus, the PL studies strongly support the DAP model for the yellow PL. Moreover, these results, particularly for as-grown samples with a high concentration of shallow donors, where fast and energy independent PL decay times are observed, are consistent with shallow donor - deep acceptor mechanism of the yellow PL. We point, however, that another common property of the DAP process, i.e., the shift of the PL maximum with increasing excitation intensity, is absent and that the yellow PL is in some cases asymmetric. These results suggest a more complex nature of the PL.

The red PL of cubic GaN (Fig. 1) shows several properties clearly similar to those of the yellow PL leading to a tentative assignment to a DAP process. The intensity of the red PL shows a strong temperature dependence, similar to that observed for the yellow PL in wurtzite samples. With increasing temperature the DAP PL is thermally deactivated, due to the ionization of shallow donors/acceptors participating in the emission, and is replaced by FB process. The ionization energy of shallow centres, estimated from PL measurements, is about $20 \div 40 \mathrm{meV}$, consistent with the donors ionization energy and not acceptors energy. The latter should be larger than $100 \mathrm{meV}$. The red PL is thus of the shallow donor - deep acceptor DAP origin. Moreover, we propose that deep acceptors active in the red PL are the same as those participating in the yellow PL of wurtzite GaN. This we conclude from the spectral position of the two emissions. The energy range of the red PL is lower by about $200 \mathrm{meV}$ from the position of the yellow PL. This shift is equal to the difference of the band gap energies of two phases of GaN. This indicates a very similar ionization energy of deep acceptors.

We have studied the surface morphology of GaN epilayers using the AFM, SEM and CL imaging. AFM scans show a granular microstructure of wurtzite and cubic epilayers, with grains of a submicron size. A large density of dislocations is expected at the grain boundaries. In most of materials dislocations are efficient centres of nonradiative recombination, Not surprising, strong intensity fluctuations of excitonic emissions are observed in the CL images, which reflect the granular structure of epilayers due to the quenching of the excitonic emission at the grain boundaries. However, we find that the yellow PL is only very weakly correlated with the surface morphology of GaN epilayers. This property of the yellow PL is very puzzling and is not yet understood. It was suggested that the yellow PL is either directly or indirectly related to dislocations, or is enhanced in the regions of large density of dislocations, since impurities participating in this PL are also implicated in the decoration of dislocations [5-7]. The red PL shows a similar property. In Fig. 2 we show the observed fluctuations of the relative intensity of the 


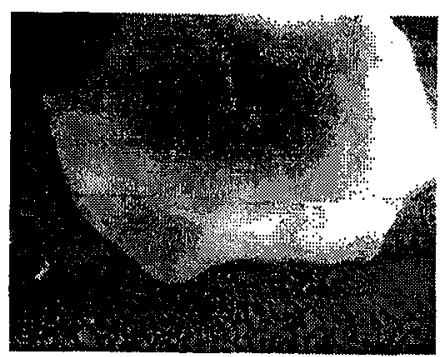

SEM image

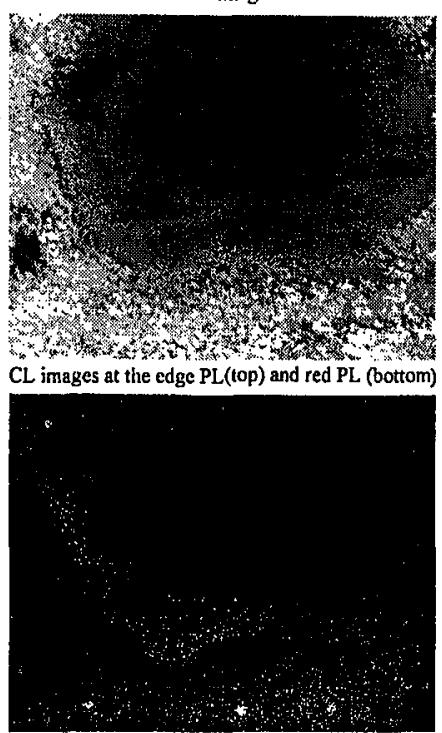

Fig. 2. Scanning electron and cathodoluminescence images at 9000 magnification of the cubic $\mathrm{GaN}$ epilayer grown by molecular beam epitaxy on (001) Si substrate. The scans were taken in the region of irregular overgrowth.

edge-to-red PLs in one of the cubic epilayers grown by MBE. Whereas the excitonic "edge" PL fluctuates in intensity and is reduced at the grains boundaries, the red PL is less affected by the surface morphology, i.e., it shows a similar behaviour to that of the yellow PL. In consequence, the red. CL dominates at the grain boundaries simply because the excitonic emission is quenched.

Concluding, the present study confirms the DAP origin of the yellow PL in wurtzite GaN and supports the shallow donor - deep acceptor mechanism of recombination. The red PL of cubic GaN is likely of the same origin. We show several analogous properties of these two PL emissions. The intensity of both yellow and red emissions is spatially homogeneous and is not reduced in the regions of high dislocations density. 
This work was partly supported by joint project no. 76568 of the Committee for Scientific Research (Poland) and Ministry of Foreign Affairs (France) and grant numbers 2P 03B 01813 and 7T 08A 00713 of the Committee for Scientific Research (Poland).

\section{References}

[1] T.L. Tansley, E.M. Goldys, M. Godlewski, B. Zhou, H.Y. Zuo, in: GaN and Related Materials, Ed. S. Pearton, Gordon and Breach Publ., Amsterdam 1997, p. 233.

[2] P. Perlin, T. Suski, M. Leszczynski, H. Teisseyre, in Ref. [1], p. 315.

[3] E.R. Glaser, T.A. Kennedy, K. Doverspike, L.B. Rowland, D.K. Gaskill, J.A. Freitas, Jr., M.A. Khan, D.T. Olson, J.N. Kuznia, D.K. Wickenden, Phys. Rev. B 51, 13326 (1995).

[4] M. Godlewski, J.P. Bergman, B. Monemar, T. Suski, I. Grzegory, S. Porowski, to be published.

[5] M. Godlewski, E.M. Goldys, M.R. Phillips, R. Langer, A. Barski, to be published.

[6] S. Christiansen, M. Albrecht, W. Dorsch, H.P. Strunk, A. Pelzmann, M. Mayer, M. Kamp, K.J. Ebeling, C. Zanottifregonara, G. Salvati, Mater. Sci. Eng. B, Solid-State Mater. Adv. Technol. 43, 296 (1997).

[7] A. Cremades, J. Piqueras, C. Xavier, T. Monteiro, E. Pereira, B.K. Meyer, D.M. Hofmann, S. Fischer, Mater. Sci. Eng. B, Solid-State Mater. Adv. Technol. 42, 230 (1996). 